\title{
Temporal Assertions with Parametrised Propositions
}

\author{
Volker Stolz <vs@iist.unu.edu> \\ United Nations University \\ Institute for Software Technology (UNU-IIST)
}

January 26, 2007

In this work, we present an extension to our previous approach to runtime verification of a single finite path against a formula in Next-free Linear-Time Logic (LTL) with free variables and quantification.

We introduce parametrised propositions that consist of a proposition name $(p, q, \ldots)$ with arity. The payload of such a proposition occurring on a trace contains values from some object domain according to its arity. In a formula, a proposition contains the appropriate amount of variables, e.g. $p(X, Y)$ or $q(Z)$.

Variables get instantiated if a proposition matches during evaluation of a trace. Multiple occurrences of the same variable are permitted and work similar to Prolog: if a variable is already bound when a proposition is evaluated, both the proposition occurring in the current state and the bound variables must match.

From our experience with J-LO, the JAVA Logical ObSERver $[2,1]$, we found it necessary to distinguish between read and write accesses to variables, based on a static analysis of the formula. Furthermore, evaluation of uninstantiated propositions had to be considered. As interpretation (through a human) of those formulae resulted difficult and error prone due to the binding semantics, in this article we introduce a special binary binding operator $\rightarrow$ that simplifies our design in the following aspects:

- simpler binding semantics

- no static analysis necessary

- more general through quantification.

The left-hand side contains a single parameterised proposition, the right-hand side a temporal parametrised formula that may refer to the variables bound in the proposition, e.g.

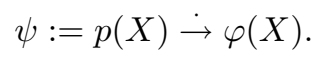

Negation is only permitted in propositional subformulae, We call the entire construct a binding expression.

Furthermore, we require that every variable occurring in a parametrised formula has previously been bound through the left-hand side of a binding operator. We can thus ensure by construction that evaluation will only encounter completely instantiated propositions, i.e. propositions, where a value for every 
variable is known. If the left-hand side does not match the current state during evaluation, the overall expression is evaluated to false.

Quantification plays a role when more than one matching proposition holds in the current state. Matching the proposition $p(X)$ against the state $\{p(1), p(3)\}$ yields two distinct bindings for variable $X: X / 1$ and $X / 3$. Quantifiers may only occur together with a parametrised proposition on the left-hand side of the binding operator. In a binding expression, all newly introduced variables through a proposition must also be quantified.

Additionally to the usual notion of LTL formulae augmented by quantified variables and bindings, we also permit predicates and functions over bound variables that can be used, for example, to compare values for inequality.

As an example, we consider the Lock-Order Reversal pattern [3], which captures a common error pattern where two processes repeatedly compete for two resources (locks), albeit in different order. This behaviour has the potential for a dead lock which can be detected by monitoring the order in which each process locks/unlocks the resources.

$$
\begin{aligned}
\Psi=\mathbf{G} & {\left[\forall t_{i} \forall l_{x}: \operatorname{lock}\left(t_{i}, l_{x}\right) \dot{\rightarrow}\left(\left[\neg \operatorname{unlock}\left(t_{i}, l_{x}\right) \mathbf{U} \exists l_{z^{\prime}}: \operatorname{lock}\left(t_{i}, l_{z^{\prime}}\right) \dot{\rightarrow} l_{z^{\prime}} \neq l_{x}\right]\right.\right.} \\
\rightarrow & \neg \operatorname{unlock}\left(t_{i}, l_{x}\right) \mathbf{U} \exists l_{z}: \operatorname{lock}\left(t_{i}, l_{z}\right) \stackrel{\rightarrow}{\rightarrow}\left[l_{z} \neq l_{x}\right. \\
& \wedge \forall l_{y}: \operatorname{lock}\left(t_{i}, l_{y}\right) \dot{\rightarrow}\left(l_{y} \neq l_{x} \wedge G \neg\left(\exists t_{j}: \operatorname{lock}\left(t_{j}, l_{y}\right) \dot{\rightarrow}\left[t_{i} \neq t_{j}\right.\right.\right. \\
& \left.\left.\left.\left.\left.\left.\wedge\left(\neg \operatorname{unlock}\left(t_{j}, l_{y}\right) \mathbf{U} \operatorname{lock}\left(t_{j}, l_{x}\right)\right)\right]\right)\right)\right]\right)\right]
\end{aligned}
$$

lock and unlock are binary propositions, binding a thread- and a lock-id, $\neq$ is a predicate.

A declarative semantics is given by expanding quantified variables through values from the finite object domain and combining them through conjunction or disjunction according to the quantifier. Operationally, evaluation of such a Temporal Assertion proceeds by means of a variant of Alternating Finite Automata, augemented with a dictionary to maintain the current bindings for each subformula. For runtime verification, we give an algorithm based on sets in disjunctive normal form that traverses the automaton in a breadth-first fashion which requires processing each state in a path exactly once and in order. It is thus suitable for online checking where an error should be detected immediately.

\section{References}

[1] E. Bodden and V. Stolz. Tracechecks: Defining semantic interfaces with temporal logic. In W. Löwe and M. Südholt, editors, 5th International Symposium on Software Composition (SC'06). To be published in Lecture Notes in Computer Science, Springer, 2006.

[2] V. Stolz and E. Bodden. Temporal Assertions using AspectJ. In H. Barringer, B. Finkbeiner, Y. Gurevich, and H. Sipma, editors, Fifth Workshop on Runtime Verification (RV'05), volume 144 of Electr. Notes in Theor. Comput. Sci. Elsevier, 2005.

[3] V. Stolz and F. Huch. Runtime Verification of Concurrent Haskell Programms. In K. Havelund and G. Roşu, editors, Proceedings of the Fourth Workshop on Runtime Verification (RV'04), volume 113 of Electr. Notes in Theor. Comput. Sci., pages 201-216. Elsevier, 2005. 